\title{
Transvesical natural orifice transluminal endoscopic surgery (NOTES) nephrectomy with kidney morcellation: a proof of concept study
}

\author{
Estevao Lima* ${ }^{*+}$ Frederico Branco*, Joana Parente*, Riccardo Autorino ${ }^{* \neq}$ \\ and Jorge Correia-Pinto*\$
}

*Life and Health Sciences Research Institute (ICVS), ICVS/3B's - PT Government Associate Laboratory,

Braga/Guimarães, ${ }^{+}$Department of Urology, Hospital de Braga, Braga, Portugal, ${ }^{*}$ Glickman Urological and Kidney

Institute, Cleveland Clinic, Cleveland, OH, USA, and ${ }^{\S}$ Department of Pediatric Surgery, Hospital de Braga, Braga,

Portugal

Accepted for publication 9 August 2011

Study Type - Therapy (case series)

Level of Evidence 4

\section{OBJECTIVE}

- To show, in a porcine model, the feasibility of a complete transvesical natural orifice transluminal endoscopic surgery (NOTES) nephrectomy with kidney extraction after morcellation through the same port.

\section{MATERIALS AND METHODS}

- Transvesical nephrectomy and morcellation were performed in six pigs at Minho University, Braga, Portugal after institutional review board approval.

- The transvesical port and the cystotomy were created under the guidance of a ureteroscope, while the remaining steps were done under the guidance of an operating telescope.

- Dissection of the renal vessels and kidney was performed using dissection

What's known on the subject? and What does the study add?

Until now, the transvaginal approach has been the only method of removing larger specimens from the abdominal cavity using natural orifice transluminal endoscopic surgery. There has been no means of extracting larger specimens in men and the means are restricted even in women, particularly in young women.

The present study shows that the difficulty of large specimen retrieval can be overcome, irrespective of the diameter of the chosen port, through natural orifices using morcellation.

grasping forceps and a vessel sealing system (LigaSure ${ }^{\mathrm{TM}}$; Covidien, Mansfield, MA, USA) and morcellation was done using a Piranha ${ }^{\mathrm{TM}}$ morcellator (Richard Wolf, Knittlingen, Germany).

\section{RESULTS}

- There were no complications related to the creation of transvesical access.

- The image provided by the telescope was superior to that of the ureteroscope, especially underwater. Morcellation was quick and effective, with the support of a fixing needle through the abdominal wall, designed to fix the kidney, after laceration of a bowel loop occurred in the first experiment
- It was found that technical improvements are needed to ensure safety of NOTES morcellation.

\section{CONCLUSIONS}

- Kidney morcellation after nephrectomy, using a natural orifice exclusively, is feasible.

- Despite technical limitations, this proof of concept study can be regarded as a potential step towards the application of NOTES in urology.

\section{KEYWORDS}

transvesical, endoscopy, kidney, nephrectomy, morcellation

\section{INTRODUCTION}

Natural orifice transluminal endoscopic surgery (NOTES) is one of the most interesting recent developments in surgery [1-3]. The main aim of this new technique is to perform surgical procedures through natural orifices and thus obtain benefits, such as improved cosmesis, owing to the lack of surgical incision in the abdominal wall. The lack of incision can also potentially be translated into lower risk of wound infection and less postoperative pain [1-3].

To date, NOTES has been successfully completed experimentally in the abdominal cavity using different methods. In 2002, Gettman et al. [4] described the first experimental application of NOTES when transvaginal nephrectomy was performed in a porcine model. Kalloo et al. [5] later reported the first natural orifice endoscopic surgery using a transluminal gastric (transgastric) port also in a porcine model; 
FIG. 1. Schematic illustraction of transvesical access to the peritoneal cavity and the selected kidney. 1, kidney; 2, operating telescope; 3, bladder.

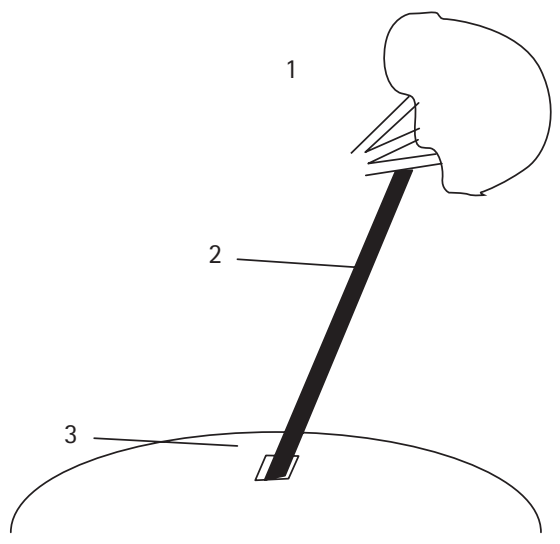

however, several limitations of performing NOTES procedures using an isolated transgastric port were subsequently pointed out [6]. During the same period, Pai et al. [7] described the use of a transluminal colonic (transcolonic) port, which was hindered by limitations related to the lack of sterility and an effective closure method.

Recently, the advantages of transvaginal access have been recognized, including the possibility of specimen extraction $[8,9]$, the safety of closure under direct vision and the in-line direct visualization of upper tract urological organs. These have allowed a progression towards the clinical application of the transvaginal port. Some recent studies have described hybrid NOTES nephrectomy [10-14] and now pure NOTES nephrectomy has also been reported [15]. An obvious limitation for the transvaginal route, however, is the fact that it is gender-specific and is not applicable in young patients [16]. The search, therefore, continues for universal NOTES access that allows the completion of a procedure and specimen retrieval from the abdominal cavity.

Our group was the first to describe the transluminal vesical (transvesical) port for NOTES applications [17]. This technique has the advantage of safe and effective access to the abdominal cavity, as the transvesical port is naturally sterile and located in the most anterior compartment of the lower abdomen. By developing the concept of combining two transluminal ports (transvesical and transgastric), we also showed the feasibility of porcine
A

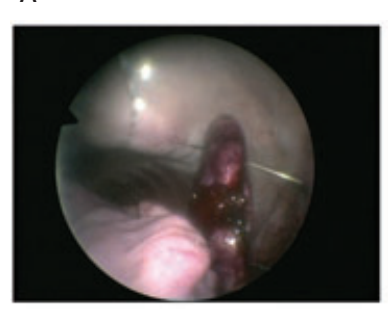

B

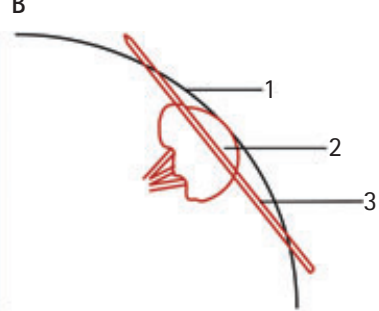

FIG. 2.

Kidney fixed to the ventral abdominal wall. $A$, intraoperative endoscopic image; $B$, schematic illustration. 1, ventral abdominal wall; 2, kidney; 3, fixing needle. nephrectomy. At that time we were not able to provide a solution for kidney extraction at the end of the intervention as the urethra diameter was a limiting factor for specimen retrieval [18].

The purpose of the present study was to show, in a porcine model, the feasibility of transvesical morcellation of a large organ, i.e. the kidney, after nephrectomy using only a single transvesical port.

\section{MATERIALS AND METHODS}

The study was conducted at Minho University, Braga, Portugal, after formal approval by our institutional review board. Six female pigs (Sus scrofus domesticus) weighing $25-30 \mathrm{~kg}$ were used. The pigs were killed at the end of the experiment and necropsy was performed in those pigs in which lesions of adjacent organs were suspected.

\section{PIG PREPARATION}

The pigs were given a liquid diet during the 3 days preceding the surgical procedure; $24 \mathrm{~h}$ before the experiment they were given no food and, during the last $6 \mathrm{~h}$ of this period, they were given no water. All procedures were performed under general anaesthesia.

\section{TRANSVESICAL ACCESS}

To establish the transvesical port, a ureteroscope was introduced through the urogenital sinus and urethra into the bladder with $\mathrm{CO}_{2}$ distention. By using $5-\mathrm{Fr}$ scissors (Model 27424 U; Karl Storz, Tuttlingen, Germany) introduced through the ureteroscope working channel, a cystotomy was performed in the bladder dome. The ureteroscope was introduced into the peritoneal cavity and a guidewire was placed through the working channel (Fig. 1).
After ureteroscope extraction, the guidewire was used to perform a $10-\mathrm{mm}$ dilatation of the urethra with a dilator designed for this experiment. The urethral dilatation allowed the introduction of a 10-mm operating telescope for single puncture surgery (Model 26034 AA; Karl Storz) in the peritoneal cavity. The entire cavity was examined.

\section{NEPHRECTOMY}

Animals were placed in the lateral decubitus position to expose the contralateral kidney. The selected kidney and its hilum were then identified by telescopic image. Mobilization of the kidney was initiated by opening the parietal peritoneum at the inferior pole of the kidney, using 5-mm scissors (Model 34321 MW; Karl Storz) introduced through the working channel of the operating telescope. The peritoneum was reflected, the hilum was exposed and the renal artery and vein were isolated from adjacent structures.

After complete dissection of the renal vessels, cauterization dissecting and grasping forceps were introduced (Model $33321 \mathrm{ML}$; Karl Storz) and a ligation device (LigaSure $^{\mathrm{TM}}$; Covidien, Mansfield, MA, USA) was used for individual division of the artery and renal vein. Kidney dissection was completed with the division of the ureter followed by isolation and mobilization of the kidney's lateral fixation and upper pole.

After releasing the organ, the renal area was inspected for bleeding.

\section{MORCELLATION}

After nephrectomy, the peritoneal cavity was emptied of $\mathrm{CO}_{2}$ and replaced with saline solution ( $2 \mathrm{~L}$ ). Before emptying $\mathrm{CO}_{2}$, the kidney was fixed to the abdominal wall with a fixing needle, created specifically for this purpose (Fig. 2). 
FIG. 3. Morcellation. $A$, Morcellator approaches the kidney; $B$, start of morcellation; $C$, $D$, end of morcellation: kidney almost unstructured. The fixing needle can be seen.
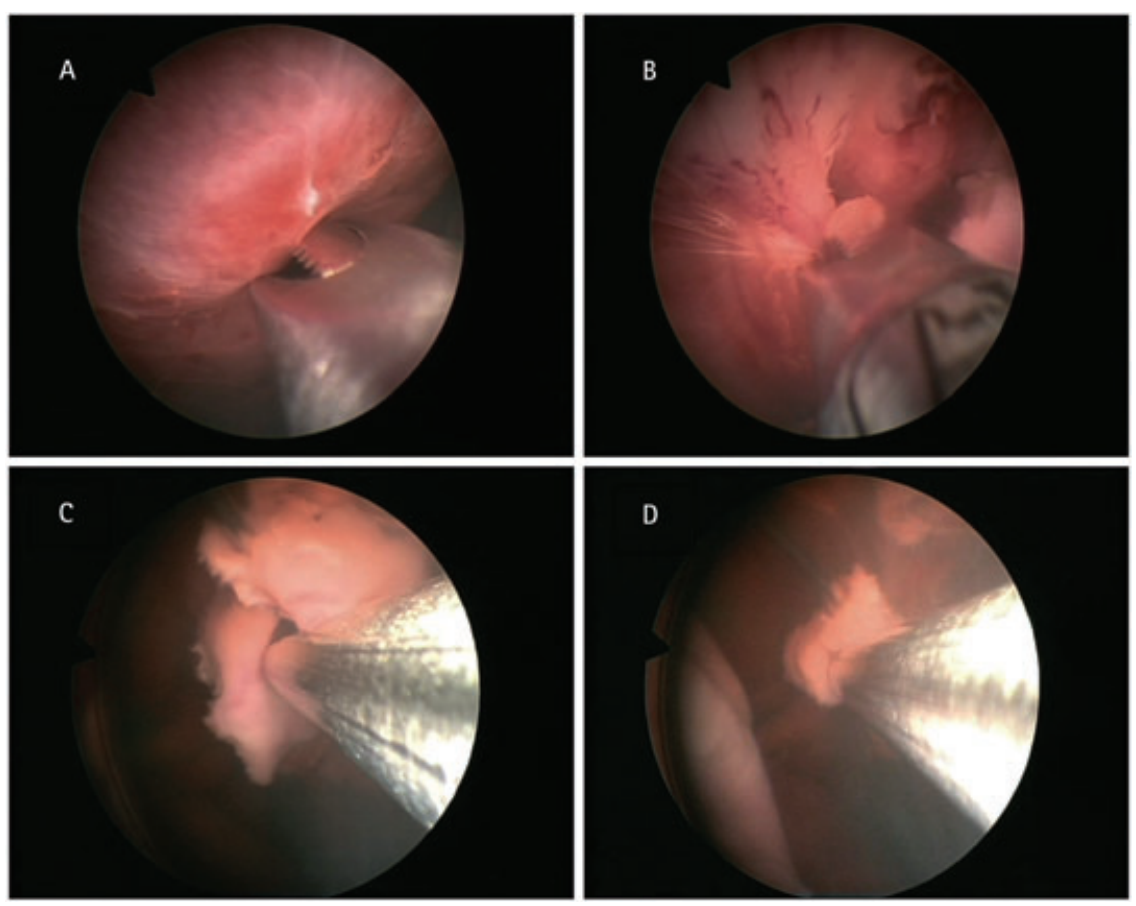

At this point, the morcellator (Piranha ${ }^{\mathrm{TM}}$, Richard Wolf, Knittlingen, Germany) was introduced through the working channel of the telescope, into the peritoneal cavity and kidney morcellation began under saline solution and was completed with the remaining saline aspirated (Fig. 3).

At the end of the procedure, the adjacent organs were inspected for any damage.

\section{RESULTS}

Nephrectomy by pure transvesical port was successfully performed in all cases. The creation of transvesical access was simple and quick, with no complications.

The introduction of the telescope into the peritoneal cavity was comfortably achieved after using the purpose-built dilator. Two cases of laceration of the urethra occurred, but these did not affect the completion of the procedure.

The image provided by the telescope was excellent, allowing us a clear view of all structures of the abdomen, and particularly the hilum structures. The kidney and its hilum dissection were easily accomplished by using $5-\mathrm{mm}$ instruments introduced through the working channel of the surgical telescope. Division of renal vessels was effectively accomplished in all cases without evidence of bleeding. After ligation of the renal vessels, the kidney was easily dissected and released from adjacent structures.

The median (range) duration of nephrectomy was 70 (45-90) min. In the first experiment, the pneumoperitoneum was not totally emptied during the filling of the peritoneal cavity with saline solution, causing a rectal prolapse. In the subsequent cases, this complication did not occur. The image provided by the telescope under saline solution was superior to that provided by gas, except when minor bleeding occurred. Kidney fixation was straightforward and allowed firm setting of the organ to the ventral abdominal wall.

Transvesical morcellation proved to be effective, allowing rapid excision of the entire kidney (median [range] time taken: 15 [10-20] min). During the first experiment, bowel laceration was suspected. This did not occur in the following experiments after we decided to fix the kidney in a more ventral position in the abdominal wall. The morcellator works only in liquid and separates morcellated tissue from irrigation fluid.

In the necropsy performed on the first pig, we found a colon laceration of $\approx 1 \mathrm{~cm}$ in size.

\section{DISCUSSION}

The present study shows the feasibility of extracting a large organ, the kidney, after morcellation by transvesical port after a pure NOTES nephrectomy. This project is part of a sequence of studies on experimental NOTES nephrectomies. The first attempt to perform a NOTES porcine nephrectomy was made by Gettman et al. [4] in 2002. Interestingly, after only 5 years, our group reported the feasibility of NOTES porcine nephrectomy by combining transvesical and transgastric ports [18]. At that time we could not provide a solution for the extraction of the kidney at the end of the intervention. In the same year, Clayman et al. [19] described a hybrid NOTES transvaginal nephrectomy in pigs, using a purpose-built surgical platform (Transport multi-lumen operating platform, USGI Medical, San Clemente, CA, USA) and a 12-mm transabdominal port. More recently, Isariyawongse et al. [20] used the combination of transgastric and transvaginal ports to perform NOTES nephrectomy with specimen retrieval through the transvaginal port.

In the clinical field, the transvaginal route for NOTES has received great attention, with several groups of investigators reporting their experience in NOTES kidney surgery. Branco et al. [10] first attempted to carry out a NOTES nephrectomy in humans by using a hybrid technique with two additional transabdominal trocars. Using this same principle, other authors have reported their clinical experiences [11-14]. In 2009, Kaouk et al. [15] were the first to report the 'pure' transvaginal NOTES nephrectomy of an atrophic kidney in a woman.

With regard to the surgical technique used in the present study, transvesical access through a ureteroscope was similar to that described previously $[5,17,18,21,22]$; however, 10-mm dilatation with a purposebuilt dilator was required for the 
introduction of the operating telescope because the diameter of the pig urethra was much smaller than that of the rigid operating telescope. This might account for two cases of urethral laceration. This complication is not likely to happen in the human urethra as it easily holds tools up to $10 \mathrm{~mm}$ [23], but the risk of urethral stricture in transvesical procedures should nevertheless be assessed in humans.

With the introduction of the operating telescope, it was found, as expected, that the image quality provided was far superior to that of the ureteroscope; it was clearer and therefore more detailed, which helped in the identification of all structures, especially those of the hilum, which required more delicate handling. In addition to this advantage, the operating telescope allowed the introduction of 5-mm instruments, which facilitated surgical manoeuvres.

The use of the LigaSure ${ }^{\mathrm{TM}}$ device in the ligation of renal vessels was safe and effective, without cases of bleeding. It should be stressed, however, that in comparison with humans, the porcine kidney is easier to dissect and its hilum easier to isolate because it does not have a significant amount of perinephric fat tissue; therefore, it is widely recognized that clips should be used for vessel ligation in humans instead of energy devices.

An important aspect to keep in mind is the evacuation of the pneumoperitoneum before morcellation. In our first experiment, a rectal prolapse occurred because the pneumoperitoneum was not completely emptied before introducing the saline solution, causing an excessive increase in intra-abdominal pressure.

The image provided by the operating telescope in liquid was superior to that given in gas, except in cases of bleeding, but a renewal of saline solution was sufficient to restore the image quality.

Morcellation, allowing kidney extraction through the working channel of the operating telescope, was effective and speedy. An advantage was the possibility of separating the morcellated material from the irrigation fluid, which would allow histological study in certain clinical situations. The morcellator only works in liquid, which restricted its application. This

TABLE 1 Comparison of transluminal ports

\begin{tabular}{lllll} 
& Stomach & Bladder & Vagina & Colon \\
\hline Morcellation & - & Yes & - & - \\
Larger intact specimen retrieval & No & No & Yes & Yes \\
Small intact specimen retrieval & Yes & No & Yes & Yes
\end{tabular}

limitation needs to be overcome by the creation of an instrument able to operate in gas. If, on the one hand, the speed and suction of morcellation represent an advantage in terms of operating time, it can be also regarded, on the other hand, as a limitation. Any deviation or uncontrolled handling could result in laceration or perforation of adjacent organs, as happened in the first experiment where there was bowel loop laceration (revealed at necropsy). After this complication during the first case, the kidney was placed far from other organs. To this end, a fixing needle was designed and built. This was passed under vision through the ventral abdominal wall, then the kidney and, finally, again the ventral abdominal wall to firmly fix the kidney at a superior level compared with other organs. Thus, the probability of accidentally morcellating adjacent organs was minimized, and no further organ injuries occurred. This fixation of the kidney in the abdominal wall also facilitated morcellation by better exposing the kidney and moving it away from bowel loops, and the introduction of saline solution caused the bowel loops to move into a higher plane owing to their density and the presence of gas.

Morcellation through other natural orifices has never been reported, possibly because: 1) the transgastric port does not allow the use of rigid morcellators and, as far as we know, flexible ones do not exist; 2) the transcolonic port has an associated infection risk and the morcellator is introduced between the bowel loops; 3 ) the transvaginal port allows intact specimen retrieval, despite being restricted to females. A solution for the removal of large organs in males has not so far been reported.

The present study describes, for the first time, the morcellation of an organ within the abdominal cavity through the natural orifice of the transvesical port. This finding might help overcome the limitation commonly pointed out - the impossibility of specimen retrieval $[2,3,16,18]$. Indeed, in the future, the transvesical port might represent an ideal pathway to organ morcellation as it is naturally sterile and is situated above the bowel loops, unlike all other natural orifices (Table 1). Nevertheless, it should be recognized that the safety of morcellation through the transvesical port is still far from perfect. Technical improvement of the morcellation device is needed in the future, as well as the creation of other ancillary devices to maximize safety. In this regard, we believe that a protector bag would be necessary to carry out this procedure in order to avoid any tissue spillage that might occur during the morcellation. This is critical for the non-functioning kidney with stones or infection, or in a kidney where a tumour is present.

In conclusion, complete NOTES transvesical nephrectomy, including specimen retrieval after morcellation, is feasible in the porcine model. The present study may serve as a starting point for the development of a new generation of morcellators aiming to make transvesical morcellation of abdominal organs safe and effective.

\section{ACKNOWLEDGEMENTS}

This study was supported by the Science \& Technology Foundation (FCT), Portugal - PTDC/SAU-OSM/105578/2008. The authors acknowledge Karl Storz, Richard Wolf and Covidien for their support with equipment. Estevao Lima and Frederico Branco contributed equally to this article.

\section{CONFLICT OF INTEREST}

None declared.

\section{REFERENCES}

Gettman MT, White WM, Aron M

et al. Where do we really stand with 
LESS and NOTES? Eur Uro/ 2011; 59 :

231-4

Autorino R, Cadeddu JA, Desai MM

et al. Laparoendoscopic single-site and natural orifice transluminal endoscopic surgery in urology: a critical analysis of the literature. Eur Urol 2011; 59: 26-45

3 Lima E, Rolanda C, Autorino R, Correia-Pinto J. Experimental foundation for natural orifice transluminal endoscopic surgery and hybrid natural orifice transluminal endoscopic surgery. BJU Int 2010; 106 : 913-8

4 Gettman MT, Lotan Y, Napper CA, Cadeddu JA. Transvaginal laparoscopic nephrectomy: development and feasibility in the porcine model. Urology 2002; 59: 446-50

5 Kalloo AN, Singh VK, Jagannath SB et al. Flexible transgastric peritoneoscopy: a novel approach to diagnostic and therapeutic interventions in the peritoneal cavity. Gastrointest Endosc 2004; 60: 114-7

6 Rolanda C, Lima E, Pêgo JM et al. Third-generation cholecystectomy by natural orifices: transgastric and transvesical combined approach (with video). Gastrointest Endosc 2007; 65: 111-7

7 Pai RD, Fong DG, Bundga ME et al. Transcolonic endoscopic cholecystectomy: a NOTES survival study in a porcine model (with video). Gastrointest Endosc 2006; 64: 428-34

8 Breda G, Silvestre P, Giunta A, Xausa D, Tamai A, Gherardi L. Laparoscopic nephrectomy with vaginal delivery of the intact kidney. Eur Urol 1993; 24: $116-7$
9 Gill IS, Cherullo EE, Meraney AM, Borsuk F, Murphy DP, Falcone T. Vaginal extraction of the intact specimen following laparoscopic radical nephrectomy. J Urol 2002; 167: 238-41

10 Branco AW, Branco Filho AJ, Kondo W et al. Hybrid transvaginal nephrectomy. Eur Urol 2008; 53: 1290-4

11 Sotelo R, de Andrade R, Fernández G et al. NOTES hybrid transvaginal radical nephrectomy for tumor: stepwise progression toward a first successful clinical case. Eur Urol 2010; 57: 138-44

12 Kaouk JH, White WM, Goel RK et al. NOTES transvaginal nephrectomy: first human experience. Urology 2009; 74: 5-8

13 Alcaraz A, Peri L, Molina A et al. Feasibility of transvaginal NOTESassisted laparoscopic nephrectomy. Eur Urol 2010; 57: 233-7

14 Porpiglia F, Fiori C, Morra I, Scarpa RM. Transvaginal natural orifice transluminal endoscopic surgery-assisted minilaparoscopic nephrectomy: a step towards scarless surgery. Eur Urol 2011; 60: 862-6

15 Kaouk JH, Haber GP, Goel RK et al. Pure natural orifice translumenal endoscopic surgery (NOTES) transvaginal nephrectomy. Eur Urol 2010; 57: 723 6

16 Lima E, Rolanda C, Correia-Pinto J. NOTES performed using multiple ports of entry: current experience and potential implications for urologic applications. J Endourol 2009; 23: 759-64

17 Lima E, Rolanda C, Pêgo JM et al. Transvesical endoscopic peritoneoscopy: a novel $5 \mathrm{~mm}$ port for intra-abdominal scarless surgery. J Urol 2006; 176 : 802-5

18 Lima E, Rolanda C, Pêgo JM et al. Third-generation nephrectomy by natural orifice transluminal endoscopic surgery. J Urol 2007; 178: 2648-54

19 Clayman RV, Box GN, Abraham JB et al. Rapid communication: transvaginal single-port NOTES nephrectomy: initial laboratory experience. J Endourol 2007; 21: 640-4

20 Isariyawongse JP, McGee MF, Rosen MJ, Cherullo EE, Ponsky LE. Pure natural orifice transluminal endoscopic surgery (NOTES) nephrectomy using standard laparoscopic instruments in the porcine model. J Endourol 2008; 22: 1087-91

21 Lima E, Henriques-Coelho T, Rolanda C et al. Transvesical thoracoscopy: a natural orifice translumenal endoscopic approach for thoracic surgery. Surg Endosc 2007; 21: 854-8

22 Lima E, Rolanda C, Osório L et al. Endoscopic closure of transmural bladder wall perforations. Eur Urol 2009; 56: 151-7

23 Branco F, Pini G, Osório L et al. Transvesical peritoneoscopy with rigid scope: feasibility study in Human male cadaver. Surg Endosc 2011; 25: 2015-9

Correspondence: Estevao Lima, Life and Health Sciences Research Institute (ICVS), School of Health Sciences, University of Minho, Campus de Gualtar, 4709-057 Braga, Portugal.

e-mail: estevaolima@ecsaude.uminho.pt

Abbreviation: NOTES, natural orifice transluminal endoscopic surgery. 